\title{
Enhancement of Color Images by Scaling the DCT Coefficients and Interpolation
}

\author{
Elsa Alias \\ Department of Electronics and Communication \\ Federal Institute of Science and Technology \\ Ernakulam, India
}

\author{
Jilu George \\ Assistant Professor \\ Department of Electronics and Communication \\ Federal Institute of Science and Technology \\ Ernakulam, India
}

\begin{abstract}
This paper presents the enhancement of color images in the frequency domain with interpolation. The novelty in our approach is the treatment of the chromatic components, while previous techniques treated only the luminance components and the enhanced image is interpolated with bicubic interpolation. After interpolation, the interpolated surface is smoother than corresponding surface obtained by the enhancement. The treatment of chromatic components improves the visual quality of the images to a great extent. The proposed technique, is more efficient than the spatial domain method.
\end{abstract}

This paper investigate how we can increase the number of pixels and there by smoothening the enhanced surface.

\section{General Terms}

Image processing, Digital camera application, Scaling coefficients, Algorithms.

Keywords color image enhancement, discrete cosine transform, Y-Cb-Cr color space, DC-AC coefficients, bicubic interpolation etc..

\section{INTRODUCTION}

Image enhancement is required mostly for better visualization or rendering of images to aid our visual perception. There are various reasons, why a raw image data requires processing before display. The dynamic range of the intensity values may be small due to the presence of strong background illumination, as well as due to the insufficient lighting. It may be the other way also. Due to these problems, the scene appears to be too dark while in some other places it is too bright. An example is shown in fig. 1(a). It can be seen that the persons stand at the backside is not clear and many details are not visible. In such images it is necessary to enhance the background illumination by preserving the colour and contrast [1]. After bicubic interpolation the image surface become more smoother and the number of pixels increases. The image is very clear when the number of pixels is high. The result of such a processing with improved display is shown in fig. 1(b). The display of a color image depends upon three fundamental factors, namely 1) its brightness 2) contrast and 3 ) color. After enhancement the output is interpolated. In this work we have considered all the above attributes while designing a simple computationally efficient algorithm. The drawbacks of the existing methods are 1) it will process only the luminance part 2) all enhancement techniques are spatial domain based. 3)it enhance the brightness and preserve only the contrast.

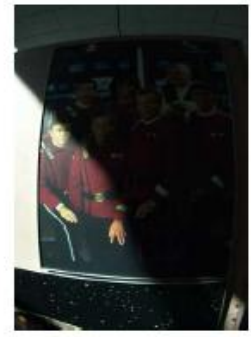

(a)

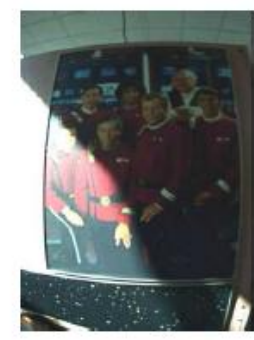

(b)
Fig 1 (a) original image and (b) bicubic interpolated image

\section{PROPOSED ALGORITHM}

The important steps used in this work are given below.

1) Read the input image.

2) Convert into $\mathrm{Y}-\mathrm{Cb}-\mathrm{Cr}$ colour space.

3) Apply DCT for all thee colour spaces.

4) Apply the scaling coefficients for all three color spaces.

4.1) For brightness $\rightarrow$ scale only DC coefficients.

4.2) For contrast $\rightarrow$ scale DC \& AC coefficients.

4.3) For color $\rightarrow$ scale $\mathrm{Cb} \& \mathrm{Cr}$ components.

5) Apply inverse DCT.

6) Convert into RGB color space.

7) Apply bicubic interpolation.

8) comparison using image quality index.

\section{1) Input image}

The input image must be a color image. Every image is on the basis of color, contrast and brightness. These three primary elements are altered in order to obtain enhanced image. Thus direct observation and recorded color images of the same scenes are often strikingly different because human visual perception computes the conscious representation with vivid color.[2],[3].

\section{2) Y-Cb-Cr color space}

The $\mathrm{Y}-\mathrm{Cb}-\mathrm{Cr}$ color space is related to the R-G-B color space as follows:

$$
\begin{aligned}
& \mathrm{Y}=0.502 \mathrm{G}+0.098 \mathrm{~B}+0.256 \mathrm{R} \\
& \mathrm{Cb}=-0.290 \mathrm{G}+0.438 \mathrm{~B}-0.148 \mathrm{R}+128 \\
& \mathrm{Cr}=-0.366 \mathrm{G}-0.071 \mathrm{~B}+0.438 \mathrm{R}+128
\end{aligned}
$$

$\mathrm{Y}-\mathrm{Cb}-\mathrm{Cr}$ is a family of color space used as a part of the color image pipeline in video and digital photography systems. $\mathrm{Y}$ is the luma component and $\mathrm{Cb}$ and $\mathrm{Cr}$ are the blue difference and red difference chroma components. The $\mathrm{Y}-\mathrm{Cb}-\mathrm{Cr}$ color space was developed as part of ITU-R BT.601 during the development of a world-wide digital component video 
standard

\section{3) Discrete Cosine Transform (DCT)}

DCT [4] is more commonly used in image compression algorithms, because it reduces the number of computational complexity. Fig 2 shows the DCT of the original image. In the case of a 2 -D image $\{x(m, n) .0 \leq m \leq N$ $1,0 \leq \mathrm{n} \leq \mathrm{N}-1\}$ it is given by

$$
\begin{gathered}
C(k, l)=\frac{2}{N} \alpha(k) \alpha(l) \sum_{m=0}^{N-1} \cdot \sum_{n=0}^{N-1}(x(m, n) \\
\cos \left\{\frac{(2 m+1) \pi k}{2 N}\right\} \cos \left\{\frac{(2 n+1) \pi l}{2 N}\right\} \\
0 \leq \mathrm{k}, 1 \leq \mathrm{N}-1
\end{gathered}
$$

Where $\alpha(p)$ is given by

$$
\alpha(p)=\left\{\begin{array}{r}
\sqrt{(1} / 2), \text { for } p=0 \\
1, \text { otherwise }
\end{array}\right.
$$

The coefficient $\mathrm{C}(0,0)$ is the DC coefficient and the remaining are the $\mathrm{AC}$ coefficient of the block. The normalized transform coefficient

$$
c^{\wedge}(\mathrm{k}, 1)=\mathrm{c}(\mathrm{k}, \mathrm{l}) / \mathrm{N}
$$

Let $\mu$ and $\sigma$ denote the mean and standard deviation of an $\mathrm{N} \times \mathrm{N}$ Image.

$$
\mu=c^{\wedge}(0,0)
$$

$$
\sigma=\sqrt{\sum_{k=0}^{N-1} \cdot \sum_{l=0}^{N-1} \mathrm{c}^{\wedge}(\mathrm{k}, \mathrm{l}) 2}-\mu 2
$$

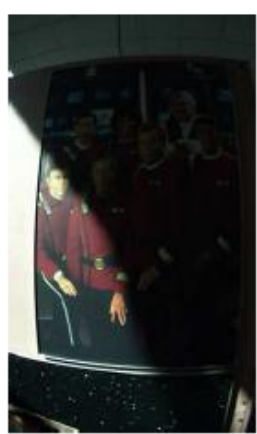

(a)

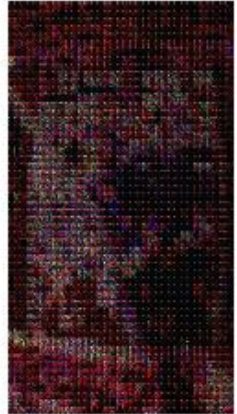

(b)
Fig 2. (a) original image and (b) DCT of image.

\subsection{1) Adjustment of local background illumination.}

In adjusting the local background illumination, the DC coefficient of a block is used. The Dc value gives the mean of the brightness distribution of the block. This adjustment may be performed by mapping the brightness values to a value in the desired range. This function should be monotonic in the given range. Let Imax be the maximum brightness value of the image Let the DCT coefficient of a $8 \times 8$ block of the luminance component $(\mathrm{Y})$ be denoted by $\{\mathrm{Y}(\mathrm{k}, 1), 0 \leq \mathrm{k}, \mathrm{l} \leq$ $7\}$.In adjusting the local brightness, the DC coefficient is mapped to $\tilde{y}^{\wedge}(0,0)$

$$
\tilde{y}^{\wedge}(0,0)=\operatorname{Imax} \cdot f\left(y^{\wedge}(0,0) / \operatorname{Imax}\right)
$$

The functions used are the twicing function [5] ,a function used in [6], and the S-function [7].

$$
\tau(x)=x(2-x), \quad 0 \leq x \leq 1
$$

$$
\eta(\mathrm{x})=\left(\mathrm{x}^{1 / \gamma}+\left(1-(1-\mathrm{x})^{1 / \gamma}\right)\right) / 2 \quad, 0 \leq \mathrm{x} \leq 1
$$

$\varphi(x)=\left\{\begin{array}{c}n\left(1-\left(1-\frac{x}{m}\right) p 1\right), 0 \leq x \leq m \\ n+(1-n)\left(\frac{x-m}{1-m}\right) p 2 \\ m \leq x \leq 1,0 \leq m \leq n \leq 1, p 1, p 2>0\end{array}\right.$

The reason for choosing the functions are

1) They have been employed earlier in developing image enhancement algorithms.

2) There is no single function which has been found to provide the best performance for every image.

Fig. 3 shows the enhanced image by scaling DC coefficients only using $ح(\mathrm{x}), \eta(\mathrm{x}), \Psi(\mathrm{x})$. It may be noted that though the dark regions get brightened by these processes, the image lack the sharpness or details of the original one. so to preserve the local contrast, apply the following simple procedure.
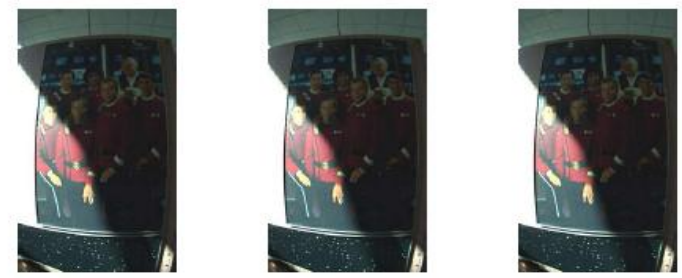

Fig.3 Enhanced image by scaling the coefficients using functions.

\section{4.2) Preservation of local contrast.}

The enhancement factor

$$
\mathrm{k}=\mathrm{f}(\mathrm{Y}(0,0)) / \mathrm{Y}(0,0)
$$


Contrast, is defined as the diference in intensity between the highest and lowest intensity levels in an image. The concept of contrast simultaneous related entirely to the perceived brightness, does not depend simply on its intensity ; they appear to the eye to become darker as the background gets lighter. The proposed image enhancement algorithm is applicable to any DCT - based image compression standard such as JPEG,MPEG.

\subsection{3) Preservation of colors.}

All the existing techniques only changes the luminance component and keep the chrominance component unaltered. Though in the $\mathrm{Y}-\mathrm{Cb}-\mathrm{Cr}$ Color space the chrominance components are decorrelated better than that in the R-G-B color space, the increasing values in the $\mathrm{Y}$ component usually tends to desaturate the colors. Typically one may observe from the conversion matrix for going from the $\mathrm{Y}-\mathrm{Cb}-\mathrm{Cr}$ space to the R-G-B space, for $\mathrm{G}>\mathrm{R}$ and $\mathrm{G}>\mathrm{B}$ increasing $\mathrm{Y}$ while keeping $\mathrm{Cb}$ and $\mathrm{Cr}$ unchanged reduces both the $\mathrm{R} / \mathrm{G}$ and $\mathrm{B} / \mathrm{G}$ factors[8]. This is the reason that the chromatic components should be also processed for preserving the colors. Let $\mathrm{U}=\{\mathrm{U}(\mathrm{k}, \mathrm{l}) \mid 0 \leq \mathrm{k}, 1 \leq(\mathrm{N}-1)\}$ and $\mathrm{V}=\{\mathrm{V}(\mathrm{k}, 1) \mid 0 \leq \mathrm{k}, 1 \leq(\mathrm{N}-1)\}$ Denote the DCT coefficients of the $\mathrm{Cb}$ and $\mathrm{Cr}$ components, respectively. If the luminance component of an image is uniformly scaled by a factor $\mathrm{k}$, the colors of the processed image with $\tilde{y}, \tilde{u}$, and $\tilde{v}$ are preserved by the following equations

$$
\tilde{\mathrm{u}}(i, j)=\left\{\begin{aligned}
N\left(k\left(\frac{U(i, j)}{N}-128\right)+128\right), & i=j=0 \\
k U(i, j), & \text { otherwise }
\end{aligned}\right.
$$

$\tilde{\mathrm{v}}(i, j)=\left\{\begin{aligned} N\left(k\left(\frac{V(i, j)}{N}-128\right)+128\right), & i=j=0 \\ k V(i, j), & \text { otherwise }\end{aligned}\right.$

\section{5) Inverse Discrete Cosine Transform (IDCT)}

The inverse DCT is given by

$$
\begin{gathered}
x(m, n)=\frac{2}{N} \sum_{k=0}^{N-1} \cdot \sum_{l=0}^{N-1} \alpha(k) \alpha(l) c(k, l) \\
\cos \left\{\frac{(2 m+1) \pi k}{2 N}\right\} \cos \left\{\frac{(2 n+1) \pi l}{2 N}\right\} \\
0 \leq \mathrm{k}, 1 \leq \mathrm{N}-1
\end{gathered}
$$

Here the coefficient $x(0,0)$ is the DC coefficient and the remaining are the $\mathrm{AC}$ coefficient for the block. Generation of many image and video compression schemes perform the Discrete Cosine Transform (DCT) to represent image data in frequency space [9]. In MPEG, the DCT is used to code interframe prediction error terms. The distribution of these coefficient is explored. Many digital image and video compression schemes use block based DCT as the transform coding. In particular JPEG and MPEG use the DCT to concentrate image information. Image compression systems often divide each image into multiple planes, one for luminance and two for color. The images are also spatially divided into blocks, usually $8 \times 8$ pixels. The DCT is applied to each block in each plane.

\section{6) R-G-B color space}

The syntax used to Convert $\mathrm{YCbCr}$ color values to RGB color space is $y=y c b c r 2 \operatorname{rgb}(a)$, where a is the input image. The RGB color model is an additive color model in which red, green, and blue light are added together in various ways to reproduce a broad array of colors. The name of the model comes from the initials of the three additive primary colors, red, green, and blue. The main purpose of the RGB color model is for the sensing, representation, and display of images in electronic systems, such as televisions and computers, though it has also been used in conventional photography. Before the electronic age, the RGB color model already had a solid theory behind it, based in human perception of colors.

\section{7) Bicubic Interpolation}

Bicubic interpolation is an extension of cubic interpolation for interpolating data points on a two dimensional regular grid. The interpolated surface is smoother than corresponding surfaces obtained by bilinear interpolation or nearest-neighbor interpolation. Bicubic interpolation[10] can be accomplished using either Lagrange polynomials, cubic splines, or cubic convolution algorithm. In image processing, bicubic interpolation is often chosen over bilinear interpolation or nearest neighbor in image resampling, when speed is not an issue. In contrast to bilinear interpolation, which only takes 4 pixels $(2 \times 2)$ into account, bicubic interpolation considers 16 pixels $(4 \times 4)$.

Images resampled with bicubic interpolation are smoother and have fewer interpolation artifacts. If the values of a function $f(\mathrm{x})$ and its derivative are known at $\mathrm{x}=0$ and $\mathrm{x}=1$, then the function can be interpolated on the interval $[0,1]$ using a third degree polynomial. This is called cubic interpolation. Bicubic interpolation is cubic interpolation in two dimensions. Bicubic interpolation is more sophisticated and produces smoother edges than bilinear interpolation. This is the method most commonly used by image editing software printer drivers and many digital cameras for resampling images. Fig 4 shows the encoding and decoding RGB information process. 


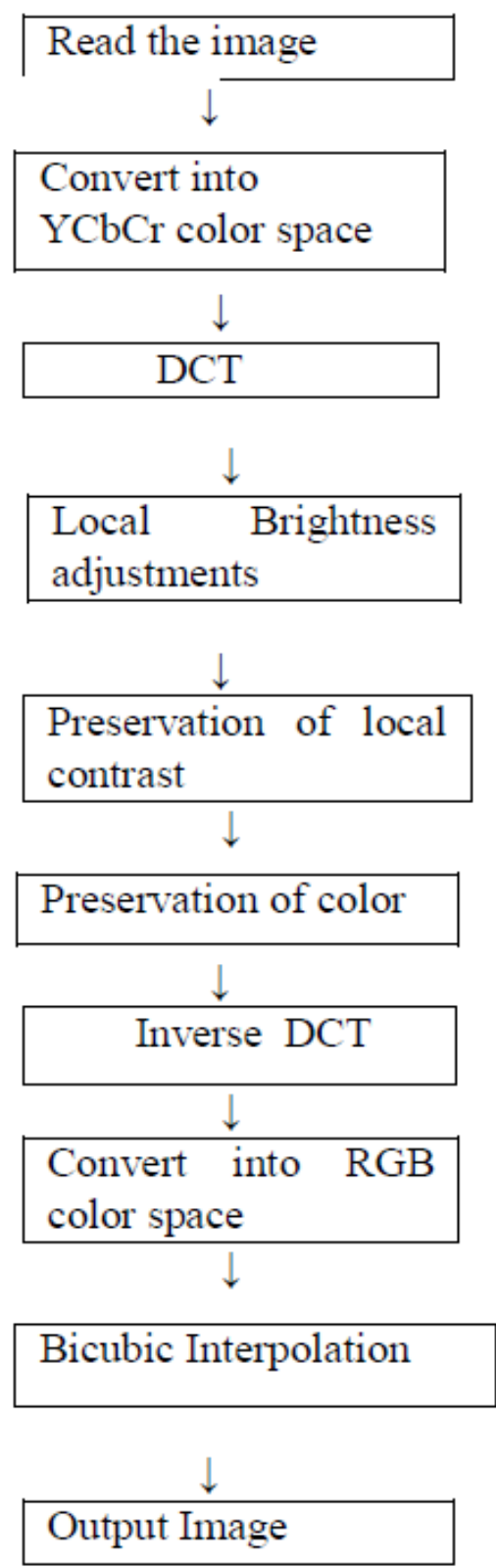

Fig .4 Encoding and Decoding RGB information process.

\section{CONCLUSION}

In this paper, we enhance the color images in the block DCT domain and after enhancement the image is interpolated with bicubic interpolation. The important feature is that it preserve the color of image by the treatment of chromatic components. The interpolation improves the visual quality of the image to a great extent. It has been found that proposed schemes outperform the existing schemes and image interpolation with RGB color space. The proposed method is applicable for Satellite and research applications, Digital camera applications. Also in this mrthod a comparison is done by using the image quality index .By analyzing using the image quality index, the interpolated image shows better $Q$ value compaired with the enhanced image. It means that the visual quality of the image is good after bicubic interpolation.

\section{COMPARISON RESULTS}

For perceptual quality evaluation purposes, we propose a new universal objective image quality index, which is easy to calculate and applicable to various image processing applications. The new index is mathematically defined and no human visual system model is explicitly employed .Let $\mathrm{x}$ and $y$ be the original and the test image signals. The proposed quality index is defined as

$$
Q=\frac{4 . \sigma \mathrm{xy} \cdot x^{-} y^{-}}{(\sigma \mathrm{x} 2+\sigma \mathrm{y} 2)\left[\left(\mathrm{x}^{-}\right) 2+\left(\mathrm{y}^{-}\right) 2\right]}
$$

Where

$$
\begin{gathered}
x^{-}=1 / N \sum_{i=1}^{N} x i \quad y^{-}=1 / N \sum_{i=1}^{N} y i \\
\sigma x 2=1 /(N-1) \sum_{i=1}^{N}\left(x i-x^{-}\right) 2 \\
\sigma y 2=1 /(N-1) \sum_{i=1}^{N}\left(y i-y^{-}\right) 2 \\
\sigma x y=1 /(N-1) \sum_{i=1}^{N}\left(x i-x^{-}\right)\left(y i-y^{-}\right)
\end{gathered}
$$

The dynamic range of $\mathrm{Q}$ is $[-1,1]$. The best value 1 is achieved if and only if $y i=x i$ for all $i=1,2 \ldots \ldots \ldots \ldots . . N$. The lowest value of -1 occurs when $y i=2 x^{-}-x i$ for all $i=$

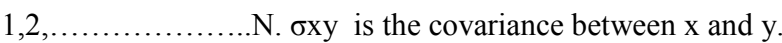
$\sigma \mathrm{x}$ and $\sigma \mathrm{y}$ are the standard deviations of $\mathrm{x}$ and $\mathrm{y}$ respectively and $\mathrm{x}^{-}$as well as $\mathrm{y}^{-}$are their respective means. Processed images with $\mathrm{Q}$ values closer to 1 are more similar in quality according to our visual perception. We have measured the $\mathrm{Q}$ values of enhanced image and the bicubic interpolated image

\begin{tabular}{|c|c|c|}
\hline Image name & $\begin{array}{l}\text { Q value } \\
\text { (without } \\
\text { interpolation) }\end{array}$ & $\begin{array}{l}\text { Q value } \\
\text { (with } \\
\text { interpolation) }\end{array}$ \\
\hline Image 2 & 0.2870 & 0.812 \\
\hline Image 7 & 0.217 & 0.910 \\
\hline Image 9 & 0.155 & 0.9842 \\
\hline Image 15 & 0.0861 & 0.893 \\
\hline Image 21 & 0.178 & 0.734 \\
\hline Image 24 & 0.0337 & 0.863 \\
\hline
\end{tabular}
for a number of images. It may be observed that the bicubic interpolated images shows better $\mathrm{Q}$ values compared with the enhanced images.

TABLE I. Performance measures of different images 
Table I shows the performance measures of different images.Image signals are generally nonstationary while image quality is often also space variant, although in practice it is usually desired to evaluate an entire image using a single overall quality value. Therefore, it is more appropriate to measure statistical features locally and then combine them together. We apply our quality measurement method to local regions using a sliding window approach. Starting from the top left corner of the image, a sliding window moves pixel by pixel horizontally and vertically through all the rows and columns of the image until the bottom -right corner is reached.

\section{ANALYSIS}

The proposed method done the bicubic interpolation with the enhanced image. After the interpolation the surface of the image become more smoother and visual quality of image also increases to a great extent. The improvement between the base paper and the new method is shown in fig. 5.After this the quality of the image is compaired by using the image quality index. By analysis the result obtained that the $Q$ value is very less for the enhanced images compaired with the interpolated images.

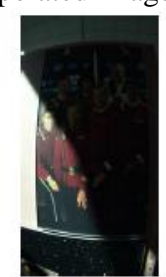

(a)

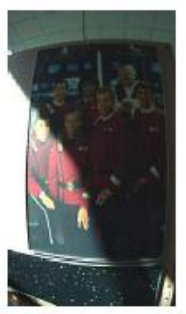

(d)

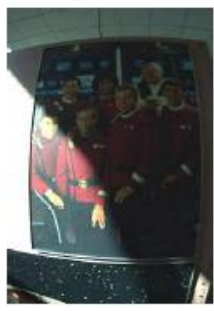

(g)

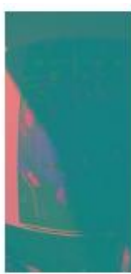

(b)

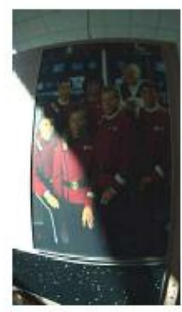

(e)

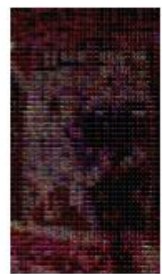

(c)

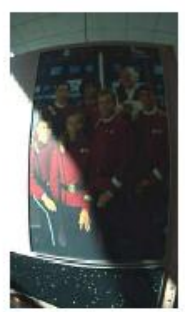

(f)

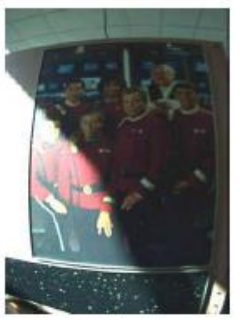

(h)
Fig.5 (a) input image (b) YCbCr image (c) DCT (d),(e),(f) background illumination using functions (g) enhanced image (h) interpolated image

\section{ENHANCED IMAGES; A FEW EXAMPLES}
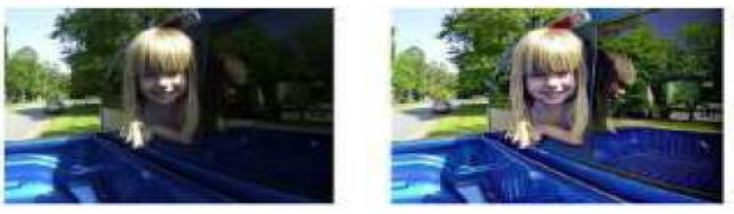

(1)

(2)

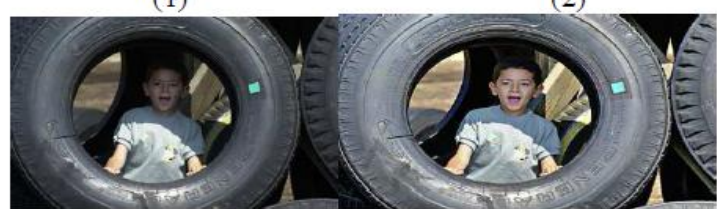

(3)

(4)

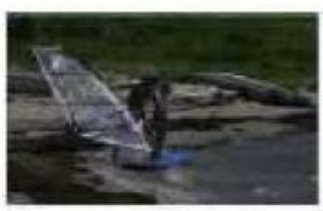

(a)

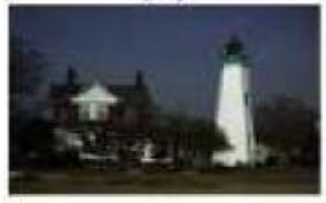

(c)

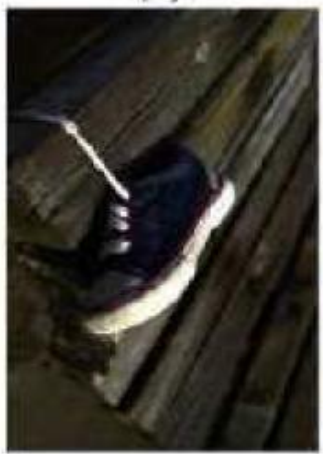

(e)

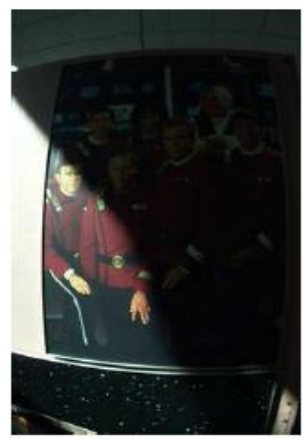

(g)

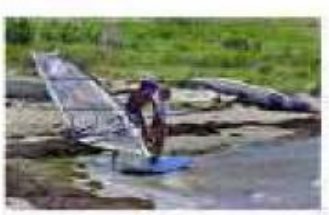

(b)

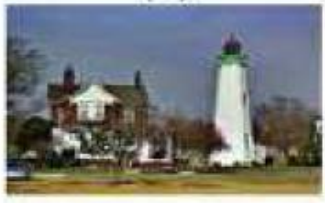

(d)

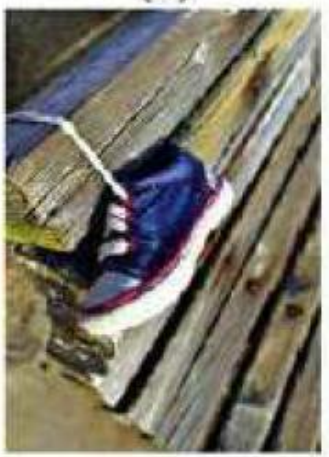

(f)

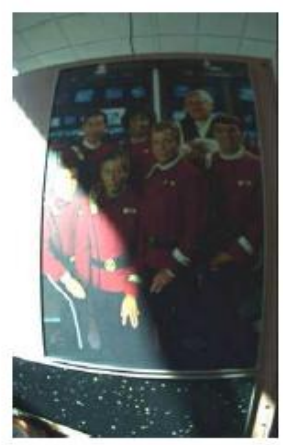

(h)
Fig 6. Few more examples of original and interpolated images (1) image 1 (2) interpolated image (3) image 2 (4) interpolated image (a) image 3 (b) interpolated image (c) image 4 (d) interpolated image. (e) image 5 (f) interpolated image. $(\mathrm{g})$ image $6(\mathrm{~h})$ interpolated image. 
Fig 6..shows the examples of original and enhanced images For observing the enhancement near edges, portions of the enhanced images near typical edges are shown in enlarged forms.

\section{REFERENCES}

[1] Jayantha Mukherjee, Sanjit K Mitra, , "enhancement of color images by scaling the DCT coefficients" ieee transaction on image processing, vol 17, no.10, October 2008.

[2] D. J. Jobson, Z. Rahman, and G. A. Woodell, "A multiscale retinex or bridging the gap between color images and the human observation of scenes," IEEE Trans. Image Process., vol. 6, no. 7, pp. 965-976, Jul. 1997.

[3] D. J. Jobson, Z. Rahman, and G. A. Woodell, "Properties and performance of a center/surround retinex," IEEE Trans. Image Process., vol. 6, no. 3, pp. 451-462, Mar. 1997.

[4] S.-H. Jung, S. K. Mitra, and D. Mukherjee, "Subband DCT: Definition, analysis and applications," IEEE Trans. Circuits Syst. Video Technol., vol. 6, no. 3, pp. 273-286, Jun. 1996.

[5] S. K. Mitra and T. H. Yu, "Transform amplitude sharpening: A new method of image enhancement," Comput. Vis. Graph. Image Process., vol. 40, pp. 205218, 1987.

[6] S. Lee, "An efficient content-based image enhancement in the domain using retinex theory," IEEE Trans. Circuits Syst. Video Technol., vol. 17, no. 2, pp. 199-213, Feb. 2007.
[7] T. K. De, “A simple programmable S-function for digital image," in Proc. 4th IEEE Region 10th Int. Conf., Bombay, India, Nov. 1989, pp. 573-576.

[8] S. K. Mitra and T. H. Yu, "Transform amplitude sharpening: A new method of image enhancement," Comput. Vis. Graph. Image Process., vol. 40, pp. 205218, 1987.

[9] P. E. Trahanias and A. N.Venetsanopoulos, "Color image enhancement through 3-D histogram equalization," in Proc. 11th IAPR Int. Conf. Pattern Recognition, The Hague, The Netherlands, Aug. 30-Sep. 3, 1992, vol. III, pp. 545-548.

[10] Akhtar P,AzharF, " A single image interpolation scheme for enhanced super resolution in bio-medical imaging", international conference on bio-informatics, New York ,2010.

[11] T. N. Pappas and R. J. Safranek, "Perceptual criteria for image quality evaluation," in Handbook of Image and Video Processing, A. C. Bovik, Ed. New York: Academic, May 2000.

[12] "Special issue on image and video quality metrics," Signal Process. vol. 70, Nov. 1998.

[13] J.-B. Martens and L. Meesters, "Image dissimilarity," Signal Process.,vol. 70, pp. 155-176, Nov. 1998.

[14] (2000, Mar.) Final report from the video quality experts group on the validation of objective models of video quality assessment. [Online].Available: http://www.vqeg.org/ 